\title{
Destruction of invariant circles
}

\author{
JOHN N. MATHER \\ Department of Mathematics, Princeton University, Princeton, NJ 08544, USA
}

\begin{abstract}
Let $\bar{f}$ be an exact, area-preserving, monotone twist diffeomorphism of the cylinder and let $\omega$ be a Liouville number. We will show that arbitrarily close to $\bar{f}$ in the $C^{\infty}$ topology there exists a $C^{\infty}$ diffeomorphism $\bar{g}$ with no homotopically non-trivial invariant circle of rotation number $\omega$.
\end{abstract}

\section{Introduction}

This paper continues our series of papers on area-preserving diffeomorphisms of the cylinder ([9] and references therein, [10] and [11]). We will prove a result about the possibility of destroying certain invariant circles of such a diffeomorphism by a $C^{\infty}$ small perturbation. Our result is a partial converse of the KAM theorem. However, we obtain such a converse only for (symplectic) mappings of a twodimensional manifold, leaving open all the questions which one could pose in higher dimensions. We also make a 'twist' hypothesis. This twist hypothesis is often satisfied in cases of interest, but, of course, one would like to remove it.

The main result of this paper is most easily formulated in terms of exact, area-preserving, monotone twist diffeormorphisms of an infinite cylinder $(\mathbb{R} / \mathbb{Z}) \times \mathbb{R}$. We will denote the set of all such diffeomorphisms by $\mathscr{T}^{1}$. The precise conditions for a diffeomorphism $\bar{f}$ of $(\mathbb{R} / \mathbb{Z}) \times \mathbb{R}$ to be a member of $\mathscr{T}^{1}$ are the conditions imposed on $\bar{f}$ in $[9, \S 2]$ and $[11, \S 2]$. We will let $\mathscr{T}^{\infty}$ denote the set of elements of $\mathscr{T}^{1}$ which are $C^{\infty}$.

For the convenience of the reader, we repeat the definition of $\mathscr{T}^{1}$ here. Let $\bar{f}$ be a mapping of $(\mathbb{R} / \mathbb{Z}) \times \mathbb{R}$ into itself. We will say that $\bar{f} \in \mathscr{T}^{1}$ if the following conditions are satisfied. First, we require that $\bar{f}$ be a $C^{1}$ diffeomorphism which maps points near the top end of the cylinder to points near the top end, and likewise for points near the bottom end. Second, setting $\bar{f}(\theta, y)=\left(\theta^{\prime}, y^{\prime}\right)$, we require that the form $y^{\prime} d \theta^{\prime}-y d \theta$ on $(\mathbb{R} / \mathbb{Z}) \times \mathbb{R}$ be exact. Third, we require that $\bar{f}$ satisfy a positive monotone twist condition, i.e. $\partial \theta^{\prime} / \partial y>0$ everywhere. Fourth, we require that $\bar{f}$ twist the cylinder infinitely at either end. To express this condition, we use a lift $f$ of $\bar{f}$ to the universal cover $\mathbb{R}^{2}$ of $(\mathbb{R} / \mathbb{Z}) \times \mathbb{R}$, and set $f(x, y)=\left(x^{\prime}, y^{\prime}\right)$. The fourth condition means that for fixed $x$ we have $x^{\prime} \rightarrow+\infty$ as $y \rightarrow+\infty$ and $x^{\prime} \rightarrow-\infty$ as $y \rightarrow-\infty$.

We will use the word 'circle' in the topological sense, i.e. a subset $\Gamma$ of $(\mathbb{R} / \mathbb{Z}) \times \mathbb{R}$ will be said to be a circle if it is homeomorphic to $\mathbb{R} / \mathbb{Z}$. We will say that $\Gamma$ is homotopically trivial if it can be deformed to a point, i.e. there is a continuous mapping $F: \Gamma \times[0,1] \rightarrow(\mathbb{R} / \mathbb{Z}) \times \mathbb{R}$ such that $F \mid \Gamma \times 0=$ inclusion and $F \mid \Gamma \times 1$ is constant. Otherwise $\Gamma$ will be said to be homotopically non-trivial. Intuitively, the homotopically non-trivial circles are the ones which 'go around' the cylinder. 
According to a theorem of Birkhoff $[2, \S 3]$, if $\Gamma$ is a homotopically non-trivial circle in $(\mathbb{R} / \mathbb{Z}) \times \mathbb{R}$ which is also $\bar{f}$-invariant for some $\bar{f} \in \mathscr{T}^{1}$, then $\Gamma$ is the graph of a Lipschitz function $u: \mathbb{R} / \mathbb{Z} \rightarrow \mathbb{R}$. See [7] for a discussion of this result and [5] and [4] for a proof. Poincaré defined the rotation number of such a circle as follows. Let $f$ be a lift of $\bar{f}$ to the universal cover $\mathbb{R}^{2}$ of $(\mathbb{R} / \mathbb{Z}) \times \mathbb{R}$. Let $\tilde{\Gamma}$ be the inverse image of $\Gamma$ under the projection $\mathbb{R}^{2} \rightarrow(\mathbb{R} / \mathbb{Z}) \times \mathbb{R}$. Let $(x, y) \in \Gamma$ and set $\left(x_{n}, y_{n}\right)=f^{n}(x, y)$. Poincaré's rotation number is defined as

$$
\rho(f, \Gamma)=\lim _{n \rightarrow \pm \infty} x_{n} / n \text {. }
$$

It is well known and easily verified that this limit exists and is independent of $(x, y)$. Birkhoff $[2, \S 4]$ proved that if $\rho$ is irrational, then there is at most one homotopically non-trivial $\bar{f}$-invariant circle of rotation number $\rho$. The question of when there do or do not exist invariant circles is very complicated and has led to deep studies by Rüssmann, Herman and others. See Herman's two volumes [5, 6] and the references therein. See also the article of Bullett [3] for some remarkable results about the 'piecewise standard map'.

In particular, the question of persistence of such invariant circles has been much studied. It has been known since the work of Poincaré in the 19th century that a generic $\bar{f} \in \mathscr{T}^{\infty}$ has no homotopically non-trivial invariant circles of rational rotation number. The big breakthrough came in the 1960s with the development of KAM (Kolmogorov, Arnold, Moser) theory. This theory deals with much more general situations than area-preserving mappings. But, for the situation we are considering here, it implies the following. Let $\bar{f} \in \mathscr{T}^{\infty}$ and let $\Gamma$ be a homotopically non-trivial $\bar{f}$-invariant circle. Let $\omega=\rho(f, \Gamma)$ be its rotation number. We say that $\omega$ satisfies a Diophantine condition, or $\omega \in \mathrm{DC}$, if there exist positive numbers $C, N$ such that

$$
|q \omega-p|>C q^{-N}
$$

for all $q, p \in \mathbb{Z} \backslash\{0\}$. According to KAM theory, if $\Gamma$ is $C^{\infty}$ and $\rho(f, \Gamma) \in \mathrm{DC}$, then there exists a neighbourhood $N$ of $\bar{f}$ in $\mathscr{T}^{\infty}$ such that if $\bar{g} \in N$ and $g$ is its lift to the universal cover (near $f$ ), then there exists a homotopically non-trivial $\overline{\boldsymbol{g}}$-invariant circle $\Lambda$ with $\rho(g, \Lambda)=\rho(f, \Gamma)$. This result is due to Moser [12], who also gave a beautiful exposition [13] of this and related results in classical dynamics. For the case of invariant circles of area-preserving mappings, improvements in the original KAM results (in the sense of requiring less differentiability) have been made by Rüssmann and Herman. The best results and a complete survey of the literature in this direction are contained in [5] and [6]. Salamon [14] gives proofs of the KAM theorem in all dimensions with careful attention to the differentiability class.

The main result of this paper is a partial converse of the KAM result on persistence of invariant circles. A real number $\omega$ is called a Liouville number if it is irrational but does not satisfy a Diophantine condition. In other words, an irrational $\omega$ is Liouville if for all positive numbers $C, N$ there exist $q, p \in \mathbb{Z} \backslash\{0\}$ such that

$$
|q \omega-p|<C q^{-N} \text {. }
$$

The main result of this paper (theorem 2.1 ) is that if $\omega$ is rational or Liouville, then 
any $\bar{f} \in \mathscr{T}^{\infty}$ can be arbitrarily well approximated in the $C^{\infty}$ topology by a $\bar{g} \in \mathscr{T}^{\infty}$ which has no invariant circle of rotation number $\omega$. For the case of rational rotation number, this is a result essentially due to Poincaré. The precise formulation we have given uses a notion ( $C^{\infty}$ topology) which was introduced after the time of Poincaré. A modern rigorous account of Poincaré's result (in the formulation we have just given) may be found in [15]. However, a method which is more quantitative than Poincaré's is necessary to obtain this result for the case of a Liouville number.

Our method is based on a study of 'Peierls's barrier', defined e.g. in [11]. Suppose $\omega$ is a rotation number, or one of $p / q+, p / q$ or $p / q-$, where $p / q$ is a rational number. Let $\bar{f} \in \mathscr{T}^{1}$ (denoted $J$ in [11]) and let $h$ be the generating function of the lift $f$ of $\bar{f}$ to the universal cover $\mathbb{R}^{2}$ of $(\mathbb{R} / \mathbb{Z}) \times \mathbb{R}$. The generating function $h$ was defined e.g. in [11, § 2]. It is uniquely characterized by the condition $f(x, y)=\left(x^{\prime}, y^{\prime}\right)$ if and only if $y=-\partial_{1} h\left(x, x^{\prime}\right)$ and $y^{\prime}=\partial_{2} h\left(x, x^{\prime}\right)$ for $\left(x, y, x^{\prime}, y^{\prime}\right) \in \mathbb{R}^{4}$. Peierls's barrier $\boldsymbol{P}_{\omega}=\boldsymbol{P}_{\boldsymbol{\omega}, h}=\boldsymbol{P}_{\boldsymbol{\omega}, \bar{f}}$ was defined in $[11, \S 6]$. For the moment, all we need to recall about it is that it is a non-negative real-valued function of a real variable, and $\bar{f}$ has a homotopically non-trivial invariant circle of rotation symbol $\omega$ if and only if $\boldsymbol{P}_{\omega, \bar{f}}$ vanishes identically.

It seems worth noting that the function $\boldsymbol{P}_{\omega}=\boldsymbol{P}_{\omega, h}=\boldsymbol{P}_{\omega, \bar{f}}$ is closely related to the number $\Delta W_{\omega}=\Delta W_{\omega, h}=\Delta W_{\omega, \bar{f}}$ introduced by the author in [8]. Indeed, $\Delta W_{\omega}$ is an upper bound for $P_{\omega}$, and $\bar{f}$ has an invariant circle of rotation symbol $\omega$ if and only if $\Delta W_{\omega}=0\left[8\right.$, proposition 5.2]. The continuity of $\Delta W_{\omega}$ at irrational numbers [8, proposition 5.1] suggested that proposition 5.2 of [8] might be useful as a necessary condition for the existence of invariant circles. The result of this paper bears out this hope. We show that it is possible to destroy invariant circles of Liouville rotation number $\omega$ by a small perturbation by showing it is possible to increase $P_{\omega}(\xi)$ by such a perturbation.

We do this by increasing $P_{p / q \pm}(\xi)$ by a perturbation $(\S 5)$ and then using a suitable modulus of continuity for $P_{\omega}(\xi)$ as a function of $\omega$ to apply this result to numbers which are well approximated by rationals. Indeed, the main technical difficulty is to obtain the modulus of continuity. The appropriate modulus of continuity is the content of theorem 2.2 . The proof of this modulus of continuity relies on the modulus of continuity we previously obtained in [11].

It would probably be possible to prove these results by following our original plan, suggested in [8], and using $\Delta W_{\omega}$ instead of $P_{\omega}(\xi)$. But it is technically easier to prove the appropriate moduli of continuity for $P_{\omega}(\xi)$ (as a function of $\omega$ ), so we use $P_{\omega}(\xi)$ in place of $\Delta W_{\omega}$. It seems that the moduli of continuity which we prove for $P_{\omega}(\xi)$ (as a function of $\omega$ ) should also hold for $\Delta W_{\omega}$, but the proof involves extra difficulties, which do not seem very interesting, so we limit ourselves to considering $P_{\omega}(\xi)$.

\section{Main results}

If $\bar{f} \in \mathscr{T}^{\infty}$, it has a lift $f$ to the universal cover $\mathbb{R}^{2}$ of $(\mathbb{R} / \mathbb{Z}) \times \mathbb{R}$, and any two such lifts differ by a translation $(x, y) \rightarrow(x+n, y)$, where $n \in \mathbb{Z}$. We let $\tilde{\mathscr{T}}^{\infty}$ denote the space of all such lifts of elements of $\mathscr{T}^{\infty}$ and we provide $\tilde{\mathscr{T}}^{\infty}$ with the $C^{\infty}$ topology. 
THEOREM 2.1. Suppose $\omega \in \mathbb{R} \backslash D C, \bar{f} \in \mathscr{T}^{\infty}$ and $f$ is a lift of $\bar{f}$ to $\mathbb{R}^{2}$. Then there exists $g \in \tilde{T}^{\infty}$ arbitrarily close to $f$, with respect to the $C^{\infty}$ topology, such that there is no homotopically non-trivial $\bar{g}$-invariant circle $\Gamma$ with $\rho(g, \Gamma)=\omega$. Here $\bar{g}$ denotes the unique element of $\mathscr{T}^{\infty}$ of which $\mathrm{g}$ is the lift.

Conversely, KAM theory implies that if $\omega \in \mathrm{DC}$ and $\vec{f}$ has a homotopically non-trivial $C^{\infty}$ invariant circle $\Gamma$ with $\rho(f, \Gamma)=\omega$, then so does every $g \in \tilde{T}^{\infty}$ near $f$ in the $C^{\infty}$ topology.

As mentioned in the introduction, the proof depends on a modulus of continuity for Peierls's barrier. Here is the result we need:

THEOREM 2.2. There exists a positive real number $C$ such that the following holds. Suppose $h: \mathbb{R}^{2} \rightarrow \mathbb{R}$ is continuous and satisfies $\left(\mathrm{H}_{1}\right)-\left(\mathrm{H}_{5}\right)$ and $\left(\mathrm{H}_{6 \theta}\right)$ of $[11, \S \S 3$ and 4]. If $p / q$ is a rational number (in lowest terms) and $\omega$ is a rotation symbol, then

$$
\left|P_{p / q+}(\xi)-P_{\omega}(\xi)\right| \leq C \theta\left|\omega^{*} q-p\right|
$$

in the case $\omega \geq p / q+$, and

$$
\left|P_{p / q-}(\xi)-P_{\omega}(\xi)\right| \leq C \theta\left|\omega^{*} q-p\right|
$$

in the case $\omega \leq p / q-$. Here $\omega^{*}$ is the number underlying $\omega$.

The above terminology was introduced in [11]. For the convenience of the reader, we review this terminology. The space $\mathscr{S}$ of rotation symbols is the disjoint union $\mathbb{R} \Perp(\mathbb{Q}+) \Perp(\mathbb{Q}-)$, where $\mathbb{R}$ denotes the set of real numbers and $\mathbb{Q}$ the set of rational numbers. We think of $\mathbb{Q}+$ and $\mathbb{Q}$ - as copies of $\mathbb{Q}$, disjoint from $\mathbb{Q}$ and from each other. If $p / q \in \mathbb{Q}$, we let $p / q-$ and $p / q+$ be the corresponding elements of $\mathbb{Q}-$ and of $\mathbb{Q}+$. If $\omega \in \mathbb{R}$, it is its own underlying number and $p / q$ is the underlying number of $p / q-$ or $p / q+$. We denote the underlying number of $\omega$ by $\omega^{*}$. We provide $\mathscr{S}$ with the unique total order for which $p / q-<p / q \leq p / q+$ and for which the mapping $\omega \mapsto \omega^{*}$ is weakly order-preserving.

We recall from [11, $\$ 3$ and 4$]$ the conditions imposed on $h$. It is a continuous real-valued function on $\mathbb{R}^{2}$ which satisfies the following:

$$
\begin{array}{cc}
h\left(x, x^{\prime}\right)=h\left(x+1, x^{\prime}+1\right) & \text { for all } x, x^{\prime} \in \mathbb{R} . \\
\lim _{|\xi| \rightarrow \infty} h(x, x+\xi)=+\infty & \text { uniformly in } x .
\end{array}
$$

$\left(H_{5}\right)$ There exists a positive continuous function $\rho$ on $\mathbb{R}^{2}$ such that

$$
\begin{gathered}
h\left(\xi, x^{\prime}\right)+h\left(x, \xi^{\prime}\right)-h\left(x, x^{\prime}\right)-h\left(\xi, \xi^{\prime}\right) \geq \int_{x}^{\xi} \int_{x^{\prime}}^{\xi^{\prime}} \rho \\
\left(H_{6 \theta}\right) \quad\left\{\begin{array}{lll}
x \rightarrow \theta x^{2} / 2-h\left(x, x^{\prime}\right) & \text { is convex } & \text { for any } x^{\prime}, \text { and } \\
x^{\prime} \rightarrow \theta x^{\prime 2} / 2-h\left(x, x^{\prime}\right) & \text { is convex } & \text { for any } x .
\end{array}\right.
\end{gathered}
$$

Here $\theta$ is a positive number. We say that $h$ satisfies $\left(\mathrm{H}_{6}\right)$ if there exists $\theta>0$ such that $h$ satisfies $\left(\mathrm{H}_{6 \theta}\right)$.

Note that in [11] we also considered conditions $\left(\mathrm{H}_{3}\right)$ and $\left(\mathrm{H}_{4}\right)$. However, these follow immediately from the above conditions, as we showed at the end of [11, $\$ 4]$, so we will not need to consider them here. 
Note that if $h$ is the generating function of a lift $f$ of $\bar{f} \in \mathscr{T}^{1}$, then $h$ satisfies the above conditions with $\theta=\cot \beta$, provided that $\beta$ is a uniform lower bound for the amount of twisting of $\bar{f}$, in the sense that the derivative of $\bar{f}$ or $\bar{f}^{-1}$ at any point turns vertical vector by at least $\beta$ (in the clockwise direction for $\bar{f}$ and the counterclockwise direction for $\left.\bar{f}^{-1}\right)$. The (completely elementary) verification of $\left(\mathrm{H}_{1}\right)-\left(\mathrm{H}_{5}\right)$ and $\left(\mathrm{H}_{6 \theta}\right)$ for such $h$ is carried out in [11].

Peierls's barrier $P_{\omega}=P_{\omega, h}$ defined in $[11, \S 6]$ is a non-negative function defined on $\mathbb{R}$, associated to a continuous function $h: \mathbb{R}^{2} \rightarrow \mathbb{R}$ satisfying $\left(\mathrm{H}_{1}\right)-\left(\mathrm{H}_{6}\right)$ and a rotation symbol $\omega$. Its value at a real number $\xi$ measures the extent to which $\omega$ fails to be in a 'minimal configuration of rotation symbol $\omega$ ' associated to $h$. We recall the relevant definitions from [11].

A configuration is a bi-infinite sequence $x=\left(\ldots, x_{i}, \ldots\right) \in \mathbb{R}^{Z}$. We set $h\left(x_{j}, \ldots, x_{k}\right)=\sum_{i=j}^{k-1} h\left(x_{i}, x_{i+1}\right)$. A configuration is said to be minimal (for $h$ ) if it minimizes $h\left(x_{j}, \ldots, x_{k}\right)$ subject to fixed boundary conditions for every pair of integers $j<k$. A configuration $x^{\prime}$ is said to be a translate of $x$ if there exist integers $j, k$ such that $x_{i}^{\prime}=x_{i+j}+k$ for all integers $i$. A basic result of Aubry says that the set of translates of a minimal configuration is totally ordered. More precisely, we will write $x<y$ (or $x>y$ ) to mean $x_{i}<y_{i}\left(\right.$ or $\left.x_{i}>y_{i}\right)$ for every integer $i$. Aubry's result is that if $x^{\prime}$ and $x^{\prime \prime}$ are both translates of the same minimal configuration, then one of the three conditions $x^{\prime}<x^{\prime \prime}, x^{\prime}=x^{\prime \prime}$ or $x^{\prime}>x^{\prime \prime}$ holds [1]. Aubry's result has the consequence that if $x$ is a minimal configuration, then there is a number $\omega=\rho(x)$, called the rotation number of $x$, such that if $x_{i}^{\prime}=x_{i+j}+k$, with $j>0$, then $x^{\prime}>x$ (resp. $x^{\prime}<x$ ) if $j \omega+k>0$ (resp. $j \omega+k<0$ ). When $\rho(x)$ is irrational, it is also called the rotation symbol $\tilde{\rho}(x)$ of $x$. When $\rho(x)$ is rational, say $p / q$ in lowest terms, $q>0$, then there is a further distinction which may be noticed by considering the translate $x_{i}^{\prime}=x_{i+q}-p$. In this case we define the rotation symbol $\tilde{\rho}(x)$ as follows: $\tilde{\rho}(x)=p / q+$ if $x^{\prime}>x ; \tilde{\rho}(x)=p / q$ if $x^{\prime}=x ; \tilde{\rho}(x)=p / q-$ if $x^{\prime}<x$.

Some further notations from [11] are: $\mathscr{M}$ denotes the set of all minimal configurations; $\mathcal{M}=\mathcal{M}_{\omega, h}$ the set of all minimal configurations (for $h$ ) of rotation symbol equal to $\omega$ or the number underlying $\omega$; if $x=\left(\ldots, x_{0}, \ldots\right) \in \mathbb{P}^{\mathbb{Z}}$ is a configuration, then $p r_{0}(x)=x_{0} \in \mathbb{R}$; and $A_{\omega}=p r_{0}\left(\mathcal{M}_{\omega}\right)$. It follows from Aubry's theory that $A_{\omega}$ is a closed subset of $\mathbb{R}$ and $p r_{0}: \mathscr{M}_{\omega} \rightarrow A_{\omega}$ is a homeomorphism, where $\mathcal{M}_{\omega}$ is provided with the topology induced from the product topology on $\mathbb{R}^{\mathbb{Z}}[1]$.

Peierls's barrier $\boldsymbol{P}_{\omega}(\xi)=\boldsymbol{P}_{\omega, h}(\xi)$ is defined for every real number $\xi$. If $\xi \in A_{\omega}$, then $P_{\omega}(\xi)$ is defined to be 0 . Otherwise, $\xi$ is in a complementary interval $\left(x_{0}, y_{0}\right)$ of $A_{\omega}$, where $x, y \in \mathcal{M}_{\omega}$. In this case $P_{\omega}(\xi)$ is defined as the minimum of $\sum_{i}\left(h\left(z_{i}, z_{i+1}\right)-\right.$ $\left.h\left(x_{i}, x_{i+1}\right)\right)$ taken over all configurations $z$ which satisfy $x_{i} \leq z_{i} \leq y_{i}, z_{0}=\xi$ and in the case that $\omega$ is rational of the form $p / q$ (in lowest terms); also $z_{i+q}=z_{i}+p$. In $[11, \S 6]$ it is proved that when $h$ is continuous and satisfies $\left(\mathrm{H}_{1}\right)-\left(\mathrm{H}_{6}\right)$, then $P_{\omega}(\xi)$ exists, is non-negative, vanishes only on $A_{\omega}$ and is a Lipschitz function of $\xi$ with Lipschitz constant $2 \theta$.

Theorem 2.2 will be proved in $\S 4$. Then in $\S 5$ we will show theorem 2.1 , using theorem 2.2 . 


\section{Properties of $h$}

In this section we derive various properties of $h$ under the sole hypotheses $\left(\mathrm{H}_{1}\right)-\left(\mathrm{H}_{5}\right)$ and $\left(\mathrm{H}_{6 \theta}\right)$.

First, recall from [11, $\S 4]$ that the one-sided first partial derivatives of $h$ exist and are locally of bounded variation. This is a consequence of $\left(\mathrm{H}_{6 \theta}\right)$ and the fact that the one-sided derivatives of a convex function of one variable exist and are monotone. Second, we have:

LEMMA. If $h: \mathbb{R}^{2} \rightarrow \mathbb{R}$ satisfies $\left(\mathrm{H}_{1}\right)-\left(\mathrm{H}_{5}\right)$ and $\left(\mathrm{H}_{6 \theta}\right)$, then there exists a unique Borel measure $\mu_{h}$ on $\mathbb{R}^{2}$ such that

$$
\mu_{h}\left([x, \xi] \times\left[x^{\prime}, \xi^{\prime}\right]\right)=h\left(\xi, x^{\prime}\right)+h\left(x, \xi^{\prime}\right)-h\left(x, x^{\prime}\right)-h\left(\xi, \xi^{\prime}\right)
$$

for any $x<\xi$ and $x^{\prime}<\xi^{\prime}$. Moreover, $\mu_{h}$ vanishes on every vertical or horizontal line, is positive on non-void open sets and is finite on bounded sets.

Proof. For coordinate rectangles, i.e. rectangles $R$ of the form $[x, \xi] \times\left[x^{\prime}, \xi^{\prime}\right]$ with $x<\xi$ and $x^{\prime}<\xi^{\prime}$, we define $\mu_{h}(R)$ by the above formula. It is easily verified that $\mu_{h}$ is an additive set function on coordinate rectangles, in the sense that if $R$ is a coordinate rectangle expressed as a finite union of other coordinate rectangles, $R=\bigcup_{i=1}^{k} R_{i}$, and $R_{i}$ intersects $R_{j}$ only in the boundary of $R_{i}$ and $R_{j}$ for $i \neq j$, then $\mu_{h}(R)=\sum_{i=1}^{k} \mu_{h}\left(R_{i}\right)$. The existence and uniqueness of a measure $\mu_{h}$ with these assigned values on coordinate rectangles is then an exercise in measure theory [16]. We omit the details.

The fact that $\mu_{h}$ vanishes on every vertical or horizontal line follows from the continuity of $h$. The fact that $\mu_{h}$ is positive on non-void open sets follows from $\left(\mathrm{H}_{5}\right)$. The fact that it is bounded on bounded sets is immediate from the definitions.

Third, we have, for $y<z$,

$$
\begin{aligned}
& \partial_{1} h(z \pm, z) \leq \partial_{1} h(z \pm, y) \leq \partial_{1} h(y \pm, y)+\theta(z-y), \\
& \partial_{2} h(z, z \pm) \leq \partial_{2} h(y, z \pm) \leq \partial_{2} h(y, y \pm)+\theta(z-y),
\end{aligned}
$$

by $\left(H_{5}\right)$ and $\left(H_{6 \theta}\right)$. Since $\partial_{1} h(y \pm, y)$ and $\partial_{2} h(y, y \pm)$ are periodic of period 1 , it follows that each of these functions has total variation $\leq 2 \theta$ over an interval of length 1.

Fourth, by (3.1), there are unique Borel measures $\nu_{h}^{1}, \nu_{h}^{2}$ on $\mathbb{R}$ such that

$$
\begin{aligned}
& \nu_{h}^{1}(y, z]=\theta(z-y)+\partial_{1} h(y+, y)-\partial_{1} h(z+, z), \\
& \nu_{h}^{2}(y, z]=\theta(z-y)+\partial_{2} h(y, y+)-\partial_{2} h(z, z+) .
\end{aligned}
$$

Obviously $\nu_{h}^{i}$ is invariant under the translation $y \rightarrow y+1$ and $\nu_{h}^{i}(y, y+1]=\theta$.

Fifth, we have, for $x \leq \xi$,

$$
\mu_{h}\left([x, \xi]^{2}\right) \leq(\xi-x) \nu_{h}^{i}(x, \xi), \quad i=1,2 .
$$

For $i=2$ this holds because

$$
\begin{aligned}
\mu_{h}\left([x, \xi]^{2}\right) & =h(x, \xi)-h(x, x)-(h(\xi, \xi)-h(\xi, x)) \\
& \leq \partial_{2} h(x, x+)(\xi-x)+\theta(\xi-x)^{2} / 2-\partial_{2} h(\xi, \xi-)(\xi-x)+\theta(\xi-x)^{2} / 2 \\
& =(\xi-x) \nu_{h}^{2}(x, \xi) .
\end{aligned}
$$


Here the first equation follows from the definition of $\mu_{h}$, the inequality is a consequence of $\left(\mathrm{H}_{6 \theta}\right)$, and the last equation follows from the definition of $\nu_{h}^{2}$. The case $i=1$ of (3.2) may be proved similarly.

Since $\nu_{h}^{j}(x, x+1]=\theta(j=1$ or 2$)$, any interval of length $\leq 1$ may be partitioned into $2 N$ subintervals, each of length $\leq 1 / N$ and $\nu_{h}^{j}$ measure $\leq \theta / N$, for any positive integer $N$. Consider such a partition, $x_{0}<x_{1}<\cdots<x_{2 N} \leq x_{0}+1$.

Sixth, we have, by (3.2) and the choice of partition,

$$
\sum_{i=0}^{2 N-1} \mu_{h}\left(\left[x_{i}, x_{i+1}\right]^{2}\right) \leq \sum_{i=0}^{2 N-1}\left(x_{i+1}-x_{i}\right) \nu_{h}^{j}\left(x_{i}, x_{i+1}\right) \leq 2 \theta / N
$$

Seventh, we have

$$
\sum_{i=j}^{k-1} h\left(x_{i}, x_{i+1}\right)=\sum_{i=j}^{k-1} h\left(x_{i}, x_{i}\right)+\int_{x_{j}}^{x_{k}} \partial_{2} h(y, y+) d y+\sum_{i=j}^{k-1} \mu_{h}\left(\Delta_{i}\right),
$$

where $\Delta_{i}$ is the triangle

$$
\left\{(y, z): x_{i} \leq y \leq z \leq x_{i+1}\right\} \quad \text { or } \quad\left\{(y, z): x_{i+1} \leq z \leq y \leq x_{i}\right\}
$$

according to whether $x_{i}$ or $x_{i+1}$ is greater. Since $y \mapsto \partial_{2} h\left(x_{i}, y+\right)$ is a function of bounded variation by $\left(\mathrm{H}_{6 \theta}\right)$, we have obviously

$$
h\left(x_{i}, x_{i+1}\right)=h\left(x_{i}, x_{i}\right)+\int_{x_{i}}^{x_{i+1}} \partial_{2} h\left(x_{i}, y+\right) d y .
$$

To verify (3.4), it is then enough to check

$$
\mu_{h}\left(\Delta_{i}\right)=-\int_{x_{i}}^{x_{i+1}} \partial_{2} h(y, y+) d y+\int_{x_{i}}^{x_{i+1}} \partial_{2} h\left(x_{i}, y+\right) d y .
$$

Let $S=\Delta_{i} \backslash \bigcup_{j}\left[\xi_{j}, \xi_{j+1}\right]^{2}$, where $x_{i}=\xi_{0}<\cdots<\xi_{2 N}=x_{i+1}$ is a partition of $\left[x_{i}, x_{i+1}\right]$ (Here we assume $x_{i}<x_{i+1}$. The case $x_{i+1}<x_{i}$ may be treated similarly.) From the definitions it follows that

$$
\mu_{h}(S)=-\sum_{j=0}^{2 N-1} \int_{\xi_{j}}^{\xi_{j+1}} \partial_{2} h\left(\xi_{j}, y+\right) d y+\int_{x_{i}}^{x_{i+1}} \partial_{2} h\left(x_{i}, y+\right) d y .
$$

Thus it is enough to verify that as the mesh of the partition tends to zero, $\mu_{h}(S)$ converges to $\mu_{h}\left(\Delta_{i}\right)$ and the first term on the right of the last formula tends to the first term on the right of the next to last formula. The convergence of $\mu_{h}(S)$ to $\mu_{h}\left(\Delta_{i}\right)$ follows from (3.3). The convergence of the first term on the right follows from

$$
\begin{aligned}
\partial_{2} h(y, y+) & \leq \partial_{2} h\left(\xi_{j}, y+\right) \leq \partial_{2} h\left(\xi_{j}, \xi_{j}+\right)+\theta\left(y-\xi_{j}\right) \\
& =\partial_{2} h(y, y+)+\nu_{h}^{2}\left(\xi_{j}, y\right],
\end{aligned}
$$

by (3.1) and the definition of $\nu_{h}^{2}$. The difference of the first terms on the right is then

$$
\leq \sum\left(\xi_{j+1}-\xi_{j}\right) \nu_{h}^{2}\left(\xi_{j}, \xi_{j+1}\right]
$$

which clearly tends to zero as the mesh of the partition tends to zero.

This proves (3.4). 


\section{Proof of theorem 2.2}

We will consider only the case $\omega \geq p / q+$, the case $\omega \leq p / q-$ being similar. First, using the main result of [11], we show that it is enough to consider the case when $p / q=0$ and $\omega=1 / q^{\prime}$. In other words, it is enough to show that there exists a positive number $C$ such that

$$
\left|P_{0+}(\xi)-P_{1 / q^{\prime}}(\xi)\right| \leq C \theta / q^{\prime}
$$

for any $h$ satisfying $\left(\mathrm{H}_{1}\right)-\left(\mathrm{H}_{5}\right)$ and $\left(\mathrm{H}_{6 \theta}\right)$, any positive number $q^{\prime}$ and any $\xi \in \mathbb{R}$.

The deduction of the case $\omega \geq p / q+$ of theorem 2.2 from (4.1) proceeds in three steps as follows. First, we consider the case $\omega=p / q+1 / q q^{\prime}$, where $q^{\prime}$ is a positive integer. This case may be reduced to (4.1) by use of the conjunction operation introduced in [11, $\S]$. We recall its definition: if $h_{1}$ and $h_{2}$ are two real-valued continuous functions on $\mathbb{R}^{2}$ satisfying $\left(\mathrm{H}_{2}\right)$, then we set

$$
h_{1} * h_{2}\left(x, x^{\prime}\right)=\min _{y} h_{1}(x, y)+h_{2}\left(y, x^{\prime}\right) \text {. }
$$

It is easily verified that $h_{1} * h_{2}$ is a real-valued continuous function on $\mathbb{R}^{2}$ satisfying $\left(\mathrm{H}_{2}\right)$, and that conjunction is an associative operation on this class of functions. More to the point for what we want to do, if $h_{1}$ and $h_{2}$ are continuous real-valued functions on $\mathbb{R}^{2}$ satisfying $\left(\mathrm{H}_{1}\right)-\left(\mathrm{H}_{5}\right)$ and $\left(\mathrm{H}_{6 \theta}\right)$, then $h_{1} * h_{2}$ also satisfies these conditions (with the same $\theta$ ) [11, lemma 5.3]. The proof of this is elementary but not obvious.

Let $H\left(x, x^{\prime}\right)=h^{* q}\left(x, x^{\prime}+p\right)$, where $h^{* q}=h * \cdots * h$ ( $q$ times) denotes the $q$-fold conjunction of $h$ with itself. It is easily verified that $A_{\omega, h}=A_{q \omega-p, H}$ and $P_{\omega, h}=P_{q \omega-p, H}$, as long as $\omega$ is a rotation symbol which is not a rational number, or is a rational number whose denominator is divisible by $q$. As we just remarked, [11, lemma 5.3] implies that $H$ is continuous and satisfies $\left(H_{1}\right)-\left(H_{5}\right)$ and $\left(H_{6 \theta}\right)$, with the same $\theta$. Thus we may reduce the case $\omega=p / q+1 / q q^{\prime}$ to the case $p / q=0, \omega=1 / q^{\prime}$ (i.e. 4.1 ), by replacing $h$ with $H$.

For the second step we consider the case $p / q+\leq \omega \leq(p+1) / q$. Here we use the main result (theorem 7.1 ) of [11]. Recall that it states that $\left|P_{\omega}(\xi)-P_{p / q}(\xi)\right| \leq$ $C \theta\left(q^{-1}+\left|\omega^{*} q-p\right|\right)$, where $C=1200$. We apply it, however, with $p / q$ replaced by $p / q+1 / q q^{\prime}$, where $q^{\prime}$ is the positive integer such that $p / q+1 / q q^{\prime} \leq \omega^{*} \leq$ $p / q+1 / q\left(q^{\prime}-1\right)$. Assuming theorem 2.2 for $\omega=p / q+1 / q q^{\prime}$, we may then deduce it for $p / q+\leq \omega \leq(p+1) / q$ :

$$
\begin{aligned}
\left|P_{\omega}(\xi)-P_{p / q+}(\xi)\right| & \leq\left|P_{\omega}(\xi)-P_{p / q+1 / q q^{\prime}}(\xi)\right|+\left|P_{p / q+1 / q q^{\prime}}(\xi)-P_{p / q+}(\xi)\right| \\
& \leq C \theta\left[\left(q q^{\prime}\right)^{-1}+\left|\omega^{*} q q^{\prime}-\left(p q^{\prime}+1\right)\right|\right]+C \theta / q^{\prime} \leq 4 C \theta / q^{\prime} \\
& \leq 4 C \theta\left|\omega^{*} q-p\right| .
\end{aligned}
$$

Finally, the case $p / q+\leq \omega$ follows trivially from the case $p / q+\leq \omega \leq(p+1) / q$. This shows that the case $\omega \geq p / q+$ of theorem 2.2 follows from (4.1).

In the first step above, note that even if $h$ is $C^{\infty}$ it is not generally the case that $H$ is $C^{1}$. Thus it seems that the mathematics forces us to consider non-differentiable functions. Of course we could try to avoid this reduction to the case $p / q=0$ and $\omega=1 / q^{\prime}$, and we have tried this. However, it seems that this reduction simplifies 
matters, even taking into account the fact that it forces us to consider non-differentiable functions.

The rest of this section is devoted to proving (4.1).

By definition $A_{0}$ is the set of $x \in \mathbb{R}$ for which $h(x, x)$ takes its minimum value. For simplicity we will assume until the end of this section that the minimum value of $h(x, x)$ is 0 . We may make this normalization, because we may add a constant to $h(x, x)$ without changing anything.

For each complementary interval $J=\left[J^{-}, J^{+}\right]$of $A_{0}$ we define a number $K_{J}$ as follows. Choose a minimal configuration $\left(\ldots, x_{i}, \ldots\right)$ of rotation symbol $0+$ such that $x_{i} \rightarrow J^{-}$as $i \rightarrow-\infty$ and $x_{i} \rightarrow J^{+}$as $i \rightarrow+\infty$. Theorem 5.8 of [1] asserts the existence of such a configuration. For such a configuration we set

$$
K_{J}=\sum_{i=-\infty}^{\infty} h\left(x_{i}, x_{i+1}\right) \text {. }
$$

(Note that $h\left(J^{-}, J^{-}\right)=h\left(J^{+}, J^{+}\right)=\min h(x, x)=0$.)

To show that this is well defined, we observe that we may sometimes extend (3.4) to infinite sums. For example, if $y$ is a monotone increasing configuration such that $y_{i} \rightarrow J^{ \pm}$as $i \rightarrow \pm \infty$, we have

$$
\int_{J^{-}}^{J^{+}} \partial_{2} h(y, y+) d y \leq \sum_{i=\infty}^{\infty} h\left(y_{i}, y_{i+1}\right) .
$$

Here the sum on the right is well defined, although it may be $+\infty$. This inequality follows from (3.4) because $h\left(y_{i}, y_{i}\right) \geq 0$ by our assumption that $\min h(x, x)=0$. In fact, we have the same inequality when we replace the sum on the right with the same sum over any subset of $\mathbb{Z}$ and the integral on the left by the integral over the corresponding subset of $\left[J^{-}, J^{+}\right]$. This shows that the sum on the right is well defined: the absolute value of the integral is bounded by $\int_{J^{-}}^{J^{+}}\left|\partial_{2} h(y, y+)\right| d y$, and consequently the sum of all terms on the right having negative value is bounded below by the negative of this quantity.

We will write $h(y)$ for $\sum_{i=-\infty}^{\infty} h\left(y_{i}, y_{i+1}\right)$ (when this is defined). Clearly $K_{J}=\min h(y)$, where the minimum is taken over all monotone increasing configurations which limit on $\boldsymbol{J}^{-}$and $\boldsymbol{J}^{+}$(as above). We may obtain an upper bound for $K$, by comparing it with the sequence defined by $y_{i}=J^{-}$for $i \leq 0$ and by $y_{i}=J^{+}$ for $i>0$. We obtain the second inequality below:

$$
\int_{J^{-}}^{J^{+}} \partial_{2} h(y, y+) d y \leq K_{J} \leq h\left(J^{-}, J^{+}\right)=\int_{J^{-}}^{J^{+}} \partial_{2} h(y, y+) d y+\mu_{h}\left(\Delta_{J}\right),
$$

where $\Delta_{J}$ is the triangle $\left\{(y, z): J^{-} \leq y \leq z \leq J^{+}\right\}$. The last equation follows from (3.4) and our normalization $h\left(J^{-}, J^{-}\right)=0$.

If $\xi \in \mathbb{R}$, we set $K_{J}(\xi)=K_{J}$ if $\xi \notin J+\mathbb{Z}$ and $K_{J}(\xi)=\min h(y)$ if $\xi \in J$, where the minimum is taken over all monotone increasing configurations $y$ limiting on $J^{ \pm}$, with $y_{0}=\xi$. For any integer $n$ we set $K_{J+n}(\xi)=K_{J}(\xi)$. This defines $K_{J}(\xi)$ for every complementary interval $J$ of $A_{0}$. 
Note that for $\xi \in J$ we have the following bounds on $K_{J}(\xi)$ :

$$
\begin{aligned}
\int_{J^{-}}^{J^{+}} \partial_{2} h(y, y+) d y & \leq K,(\xi) \leq h\left(J^{-}, \xi\right)+h\left(\xi, J^{+}\right) \\
& =\int_{J^{-}}^{J^{+}} \partial_{2} h(y, y+) d y+h(\xi, \xi)+\mu_{h}\left(\Delta_{\left[J^{-}, \xi\right]}\right)+\mu_{h}\left(\Delta_{\left[\xi, J^{+}\right]}\right),
\end{aligned}
$$

where for any closed interval $[a, b]$ we denote by $\Delta_{[a, b]}$ the triangle

$$
\{(y, z): a \leq y \leq z \leq b\} .
$$

These bounds may be proved in the same way as the bounds for $K_{J}$ were proved above.

We set

$$
K=\sum_{J} K_{J}, \quad K(\xi)=\sum_{J} K_{J}(\xi)
$$

where in each case the sum is taken over all complementary intervals of $A_{0}$ in an interval $[x, x+1]$ of unit length, with $x \in A_{0}$. It is easy to see, as a consequence of the bounds that we deduced on $K_{J}$, that these sums are absolutely convergent.

Next we show

$$
P_{0+}(\xi)=K(\xi)-K
$$

The only case we need consider is when $\xi \notin A_{0+}$, since otherwise both sides clearly vanish. We let $\xi_{-}$and $\xi_{+}$be as in the definition of $P_{\omega}(\xi)$ in $[11, \S 6]$, i.e. $\xi_{-}$and $\xi_{+}$ are minimal configurations of rotation symbol $0+$, and $\left(\xi_{0-}, \xi_{0+}\right)$ is the complementary interval of $\boldsymbol{A}_{0+}$ which contains $\boldsymbol{\xi}$. By definition

$$
P_{0+}(\xi)=\min \left\{G_{0+}(x): \xi_{i-} \leq x_{i} \leq \xi_{i+} \text { and } x_{0}=\xi\right\}
$$

where

$$
G_{0+}(x)=\sum_{i=-\infty}^{\infty} h\left(x_{i}, x_{i+1}\right)-h\left(\xi_{i-}, \xi_{i+1-}\right)=-K+\sum_{i=-\infty}^{\infty} h\left(x_{i}, x_{i+1}\right) .
$$

Thus, in order to prove (4.2), it is enough to prove that the configuration $x=\left(\ldots, x_{i}, \ldots\right)$ which achieves the minimum in the definition of $K_{J}(\xi)$ satisfies $\xi_{i-} \leq x_{i} \leq \xi_{i+}$. In fact, the Aubry graphs of $\xi_{-}, x$ and $\xi_{+}$do not cross. For example, the fact that the graphs of $\xi_{\text {- }}$ and $x$ do not cross is a consequence of the fact that $\xi_{-}$is minimal, the fact that $x$ is minimal, subject to the condition $x_{0}=\xi$, and the fact that $x$ and $\xi_{-}$are $\alpha$-asymptotic, since $x_{i} \rightarrow J_{-}$and $\xi_{i-} \rightarrow J_{-}$as $i \rightarrow-\infty$, as well as $\omega$-asymptotic, since $x_{i} \rightarrow J_{+}$and $\xi_{i-} \rightarrow J_{+}$as $i \rightarrow+\infty$. The proof that these conditions imply that the graphs of $\xi_{-}$and $x$ do not cross is the same as the proof of [1, lemma 3.9]. This proves (4.2).

We observed above that it is enough to prove (4.1) in order to obtain theorem 2.2. Our next step in the proof of (4.1) is to obtain an expression for $P_{1 / q^{\prime}}(\xi)$ similar to (4.2).

We set

$$
\begin{aligned}
L & =\min h\left(x_{0}, \ldots, x_{q^{\prime}}\right), \\
L(\xi) & =\min h\left(y_{0}, \ldots, y_{q^{\prime}}\right),
\end{aligned}
$$


where the first minimum is taken over all configurations $x$ which satisfy $x_{i+q^{\prime}}=x_{i}+1$ and the second is taken over all configurations which satisfy $y_{i+q^{\prime}}=y_{i}+1$ as well as $y_{0}=\xi$. An argument similar to the proof of (4.2), which we omit, shows

$$
P_{1 / q^{\prime}}(\xi)=L(\xi)-L
$$

Next we will show

$$
\begin{gathered}
K \leq L \leq K+16 \theta / q^{\prime} \\
K(\xi) \leq L(\xi) \leq K(\xi)+16 \theta / q^{\prime} .
\end{gathered}
$$

In view of (4.2) and (4.3), this will be enough to prove (4.1), with $C=16$, and thereby complete the proof of theorem 2.2 .

To prove $K \leq L$, we consider a configuration $x$ which satisfies $x_{i+q^{\prime}}=x_{i}+1$ and minimizes $h\left(x_{0}, \ldots, x_{q^{\prime}-1}\right)$ subject to this condition. For each complementary interval $J$ of $A_{0}$ we let $x^{J}$ denote the configuration defined by

$$
x_{i}^{J}=J^{-} \vee x_{i} \wedge J^{+} \text {. }
$$

We set $L_{J}=\sum_{i=-\infty}^{\infty} h\left(x_{i}^{J}, x_{i+1}^{J}\right)$. All but finitely many terms in this sum vanish, because all but finitely many terms have the form $h\left(J^{-}, J^{-}\right)=0$ or $h\left(J^{+}, J^{+}\right)=0$. Obviously $K_{J} \leq L_{J}$, so we have the first inequality below:

$$
K=\sum_{J} K_{J} \leq \sum_{J} L_{J} \leq L,
$$

where the sums are taken over all complementary intervals of $A_{0}$ in an interval $[x, x+1]$ of unit length, with $x \in A_{0}$.

The last inequality is a consequence of (3.4). In comparing $\sum_{J} L_{J}$ with $L$, we find that the contribution of the first two terms on the right of (3.4) is the same. In fact, the contribution of the second term is $\int_{x}^{x+1} \partial_{2} h(y, y+) d y$. The contribution of the first term apparently differs by a number of terms of the form $h\left(J^{-}, J^{-}\right)$or $h\left(J^{+}, J^{+}\right)$ which appear in the expression associated to $\sum_{J} L_{J}$ but not in that associated to $L$. However, these all vanish by our normalization.

The only difference is the contribution of the third term. In both cases the contribution has the form $\mu_{h}\left(\bigcup_{I} \Delta_{I}\right)$. For $L$, the $I$ are the complementary intervals to $\left\{x_{0}, \ldots, x_{q}\right\}$ in the interval $\left[x_{0}, x_{0}+1\right]$; for $\sum_{J} L_{J}$, they are the complementary intervals to $\left\{x_{0}, \ldots, x_{q}\right\} \cup A_{0}$ in the same interval. Thus the contribution of the third term to $\sum_{J} L_{J}$ is smaller than the contribution of the third term to $L$, because it is the measure of a smaller set.

This proves $K \leq L$. The proof that $K(\xi) \leq L(\xi)$ is similar.

To prove $L \leq K+16 \theta / q^{\prime}$, we consider $x \in \mathbb{R}$ which minimizes $h(x, x)$, so that, according to our normalization, $h(x, x)=0$. We let $N=\left(q^{\prime}+1\right) / 4$. If $N \geq 1$, we let $x=x_{0}<\cdots<x_{2 N}=x+1$ be a partition of $[x, x+1]$ such that

$$
\sum_{i=0}^{2 N-1} \mu_{h}\left(\left[x_{i}, x_{i+1}\right]^{2}\right) \leq 2 \theta / N
$$

Such a partition exists by (3.3). For each complementary interval $J=\left[J^{-}, J^{+}\right]$of $A_{0}$ in $[x, x+1]$, we choose a minimal configuration $\xi^{J}=\left(\ldots, \xi_{i}^{J}, \ldots\right)$ of rotation symbol $0+$ such that $\xi_{i}^{J} \rightarrow J^{-}$as $i \rightarrow-\infty$ and $\xi_{i}^{J} \rightarrow J^{+}$as $i \rightarrow+\infty$. Let $B$ denote the set 
of $y \in[x, x+1]$ such that $y \in A_{0}$ or $y$ is one of the $\xi_{i}^{J}$. We let $B^{\prime}$ be a subset of $B$ with $q^{\prime}+1$ points such that $B^{\prime}$ contains $x$ and $x+1$ and such that for $0 \leq i \leq 2 N$ we have that $B^{\prime} \cap\left[x_{i}, x_{i+1}\right]$ contains the least and greatest point of $B \cap\left[x_{i}, x_{i+1}\right]$, unless the latter is empty. Such a set clearly exists. We let $x=y_{0}<y_{1}<\cdots<y_{q^{\prime}-1}<y_{q^{\prime}}=x+1$ be the points of $B^{\prime}$ arranged in increasing order. We have

$$
\begin{aligned}
L \leq h\left(y_{0}, \ldots, y_{q^{\prime}}\right) & \leq K+\sum_{i=0}^{2 N-1} \mu_{h}\left(\left[x_{i}, x_{i+1}\right]^{2}\right) \\
& \leq K+2 \theta / N \leq K+16 \theta / q^{\prime} .
\end{aligned}
$$

Here the first inequality is an immediate consequence of the definition of $L$. The second inequality follows from (3.4), the definition of $K$ and the definition of the sequence $y_{0}, \ldots, y_{q^{\prime}}$. For, by definition of $K$ and (3.4), we have

and, by (3.4),

$$
K=\sum_{y \in B} h(y, y)+\int_{x}^{x+1} \partial_{2} h(y, y+) d y+\mu_{h}\left(\Delta_{B}\right)
$$

$$
h\left(y_{0}, \ldots, y_{q^{\prime}}\right)=\sum_{y \in B^{\prime}} h(y, y)+\int_{x}^{x+1} \partial_{2} h(y, y+) d y+\mu_{h}\left(\Delta_{B^{\prime}}\right),
$$

where $\Delta_{B}$ (resp. $\left.\Delta_{B^{\prime}}\right)=\cup \Delta_{I}$, the union being taken over all complementary intervals $I$ of $B$ (resp. $B^{\prime}$ ) in $[x, x+1]$. As usual, $\Delta_{I}=\{y \leq z: y, z \in I\}$. By our normalization, $h(y, y) \geq 0$, so $\sum_{B^{\prime}} h(y, y) \leq \sum_{B} h(y, y)$, as $B^{\prime} \subset B$. Clearly $\Delta_{B^{\prime}} \backslash \Delta_{B} \subset \bigcup_{i=0}^{2 N-1}\left[x_{i}, x_{i+1}\right]^{2}$ by definition of $B^{\prime}$, so we obtain the second inequality. The third inequality is a consequence of the choice of the partition $x=x_{0}<\cdots<x_{2 N}=x+1$ of $[x, x+1]$. The fourth inequality follows from the definition of $N$. This proves $L \leq K+16 \theta / q^{\prime}$ in the case that $N \geq 1$, i.e. $q^{\prime} \geq 3$. But, in any case,

$$
L \leq \int_{x}^{x+1} \partial_{2} h(y, y+) d y+\theta \leq K+\theta
$$

since $\mu_{h}\left([x, x+1]^{2}\right) \leq \theta$. Thus $L \leq K+16 \theta / q^{\prime}$ is true in every case.

The proof that $L(\xi) \leq K(\xi)+16 \theta / q^{\prime}$ is similar and will be omitted.

Clearly (4.1) follows from (4.2), (4.3) and (4.4). This completes the proof of theorem 2.2 .

\section{Proof of theorem 2.1}

We may assume without loss of generality that there is a uniform lower bound $\beta$ for the amount of twisting of $\bar{f}$. For, in any case, for any compact subset $K$ of the infinite cylinder, there exists $\bar{f}^{\prime} \in \mathscr{T}^{\infty}$ such that $\bar{f}\left|K=\bar{f}^{\prime}\right| K$ and such that there is such a uniform lower bound for the amount of twisting of $\bar{f}^{\prime}$. If $K$ is large enough, any invariant circle of any small perturbation of $\bar{f}$ or $\bar{f}^{\prime}$ whose rotation number is $\omega$ lies in $K$. It follows that if we can find $\bar{g}^{\prime}$ near $\bar{f}^{\prime}$ with no invariant circle $\Gamma$ satisfying $\rho(g, \Gamma)=\omega$, we can construct such a $\bar{g}$ near $\bar{f}$ by letting it agree with $\bar{g}^{\prime}$ on a sufficiently large compact set.

From now on we assume that there is such a uniform lower bound $\beta$ for the amount of twisting of $\bar{f}$. We have verified in [11, $\$ \$ 3$ and 4] that the generating function $h$ of the lift $f$ or $\bar{f}$ to the universal cover satisfies $\left(\mathrm{H}_{1}\right)-\left(\mathrm{H}_{5}\right)$ and $\left(\mathrm{H}_{6 \theta}\right)$. Therefore we have the modulus of continuity of theorem 2.2 for such $h$. 
In order to prove theorem 2.1 , it is enough to show that for every $\varepsilon>0$ and every positive integer $r$, there exists $h^{\prime \prime}$ in an $\varepsilon$-neighbourhood of $h$, with respect to the $C^{r+1}$ norm, such that the Peierls's barrier $P_{\omega}^{\prime \prime}$ for $h^{\prime \prime}$ does not vanish identically. Here we require that $h^{\prime \prime}$ satisfy the periodicity condition $h^{\prime \prime}\left(x+1, x^{\prime}+1\right)=h^{\prime \prime}\left(x, x^{\prime}\right)$, so that it is the generating function of the lift $g$ of an $\varepsilon$-perturbation $\bar{g}$ of $\bar{f}$, with respect to the $C^{r}$ norm. Clearly $h^{\prime \prime}$ satisfies $\left(H_{6 \theta^{\prime}}\right)$ with $\theta^{\prime}=\theta+1$ if $r \geq 1$ and $\varepsilon$ is small enough. Therefore we will still have the modulus of continuity of theorem 2.2 for $P^{\prime \prime}$, with $\theta$ replaced by $\theta^{\prime}$. Recall that $\bar{g}$ has a homotopically non-trivial invariant circle of rotation number $\omega$ if and only if $P_{\omega}^{\prime \prime}$ vanishes identically.

Our method of producing the perturbation $h^{\prime \prime}$ of $h$ consists of first choosing a rational number $p / q$ very close to $\omega$. This is possible because $\omega \notin D C$. Then we choose a minimal configuration $x$ of rotation symbol $p / q$ and a complementary interval $J$ of length $\geq q^{-1}$ to the set $\left\{x_{i}+j: i, j \in \mathbb{Z}\right\}$. This is possible because this set intersects each unit interval $[a, a+1)$ in exactly $q$ points. We choose a $C^{\infty}$ nonnegative function $u$ of small $C^{r+1}$ norm such that $u(t+1)=u(t)$ and $u$ has support in $\{J+i: i \in \mathbb{Z}\}$. We set $h^{\prime}\left(x, x^{\prime}\right)=h\left(x, x^{\prime}\right)+u(x)$.

Since $u$ vanishes on $\left\{x_{i}+j: i, j \in \mathbb{Z}\right\}$ and is non-negative, the Peierls's barrier $P_{p / q}^{\prime}$ associated to $h^{\prime}$ satisfies

$$
P_{p / q}^{\prime}(\xi)=P_{p / q}(\xi)+u(\xi)
$$

where $P_{p / q}$ is the Peierls's barrier associated to $h$.

We next explain how to construct $h^{\prime \prime}$ when $\omega>p / q$. We set $B^{\prime}\left(x, x^{\prime}\right)=$ $h^{\prime * q}\left(x, x^{\prime}-p\right)+$ const. Here $h^{\prime * q}$ denotes the conjunction of $h^{\prime}$ with itself $q$ times. Note that since $x$ is a minimal configuration of rotation symbol $p / q$ for $h$, it is also one for $h^{\prime}$ (by the choice of $u$ ), and hence it is a minimal configuration of rotation symbol 0 for $H^{\prime}$. We choose the constant in the definition of $H^{\prime}$ so that $H^{\prime}\left(x_{i}, x_{i}\right)=0$. Since $h^{\prime}$ satisfies $\left(\mathrm{H}_{1}\right)-\left(\mathrm{H}_{5}\right)$ and $\left(\mathrm{H}_{6 \theta^{\prime}}\right)$, so does $H^{\prime}$ by $[11$, lemma 5.3].

We may suppose $u$ was chosen so that it is positive on the interior of $J$. Then there is a minimal (for $H^{\prime}$ ) configuration $y$ of rotation symbol $0+$ such that $y_{i} \rightarrow J^{ \pm}$ as $i \rightarrow \pm \infty$, where $J^{-}<J^{+}$are the endpoints of $J$.

In order to obtain a suitable $h^{\prime \prime}$, we need a lower bound on $\max _{i}\left|y_{i+1}-y_{i}\right|$. Such a lower bound depends on choosing $u$ to be large enough. In fact, we may choose $u$, as above, with $C^{r+1}$ norm $\leq \varepsilon / 2$, such that

$$
u(\xi) \geq C_{r} \varepsilon / q^{r+1}, \quad \xi \in J^{\prime},
$$

where $J^{\prime}$ denotes the middle third of $J$, and $C_{r}$ is a constant depending only on $r$.

We consider an $i$ such that $y_{i} \in J^{\prime}$. (If no such $i$ exists, we may take the length of $J^{\prime}$ as our lower bound on $\max _{i}\left|y_{i+1}-y_{i}\right|$.) Since $H^{\prime}$ satisfies $\left(H_{1}\right)-\left(H_{5}\right)$ and $\left(H_{6 \theta^{\prime}}\right)$, we may apply the results of $\S 3$, in particular (3.4). Here we will apply (3.4) to the infinite sum $H^{\prime}(y)=\sum_{i=-\infty}^{\infty} H^{\prime}\left(y_{i}, y_{i+1}\right)$. The fact that we can do this has been justified in the discussion of the definition of $K_{J}$ in $\$ 4$. (In fact, $H^{\prime}(y)$ is $K_{J}$ for $h$ replaced by $H^{\prime}$.) We will compare $H^{\prime}(y)$ with $H^{\prime}\left(y^{\prime}\right)$, where $y^{\prime}$ is the configuration obtained from $y$ by removing $y_{i}$, i.e. $y_{j}^{\prime}=y_{j}$ for $j<i$ and $y_{j}^{\prime}=y_{j-1}$ for $j \geq i$. Since $y$ is minimal, $H^{\prime}\left(y^{\prime}\right) \geq H^{\prime}(y)$. On the other hand, by (3.4),

$$
H^{\prime}\left(y^{\prime}\right)-H^{\prime}(y)=\mu\left(\Delta\left[y_{i-1}, y_{i+1}\right]\right)-\mu\left(\Delta\left[y_{i}, y_{i+1}\right]\right)-\mu\left(\Delta\left[y_{i-1}, y_{i}\right]\right)-H^{\prime}\left(y_{i}, y_{i}\right),
$$


where $\Delta[a, b]=\{(y, z): a \leq y \leq z \leq b\}$ and $\mu=\mu_{H^{\prime}}$. Thus

$$
C_{r} \varepsilon / q^{r+1} \leq u\left(y_{i}\right) \leq H^{\prime}\left(y_{i}, y_{i}\right) \leq \mu\left(\Delta\left[y_{i-1}, y_{i+1}\right]\right) \leq \theta^{\prime}\left|y_{i+1}-y_{i-1}\right| .
$$

Here the first inequality follows from the fact that $y_{i} \in J^{\prime}$, the second inequality is a consequence of the definition of $H^{\prime}$, the third inequality follows from the above equation and the fact that $H^{\prime}\left(y^{\prime}\right) \geqslant H^{\prime}(y)$, and the last inequality is a consequence of (3.2) and the fact that $\nu_{H}^{i},(y, y+1) \leq \theta^{\prime}$. Obviously this lower bound on $\left|y_{i+1}-y_{i-1}\right|$ gives a lower bound of $C_{r}^{\prime} \varepsilon / q^{r+1}\left(\right.$ where $\left.C_{r}^{\prime}=C_{r} / 2 \theta^{\prime}\right)$ on one of $\left|y_{i+1}-y_{i}\right|$ or $\left|y_{i}-y_{i-1}\right|$. To summarize,

$$
\max _{i}\left|y_{i+1}-y_{i}\right| \geq C_{r}^{\prime} \varepsilon / q^{r+1} \text {. }
$$

Choose $i$ such that $\left|y_{i+1}-y_{i}\right| \geq C_{r}^{\prime} \varepsilon / q^{r+1}$, let $I$ denote the interval $\left[y_{i}, y_{i+1}\right]$ and let $v$ by a $C^{\infty}$ non-negative function such that $v(t+1)=v(t)$ and $v$ has support in the union of the intervals $I+i$ with $i \in \mathbb{Z}$. Since the length of $I$ is $\geq C_{r}^{\prime} \varepsilon / q^{r+1}$, we may choose $v$ so that it has $C^{r+1}$ norm $\leq \varepsilon / 2$ and so that $\max v \geq C_{r} \varepsilon\left(C_{r}^{\prime} \varepsilon / q^{r+1}\right)^{r+1}=$ $C_{r}^{\prime \prime} \varepsilon^{r+1} / q^{\gamma(r)}$, where $\gamma(r)=(r+1)^{2}$ and $C_{r}^{\prime \prime}=C_{r}\left(C_{r}^{\prime}\right)^{r+1}$. We set $h^{\prime \prime}\left(x, x^{\prime}\right)=$ $h^{\prime}\left(x, x^{\prime}\right)+v(x)$.

Since $v$ vanishes outside of the union of the intervals $I+i$ with $i \in \mathbb{Z}$, we have that the Peierls's barrier $P_{p / q^{+}}^{\prime \prime}$ associated to $h^{\prime \prime}$ satisfies

$$
P_{p / q+}^{\prime \prime}(\xi)=P_{p / q+}^{\prime}(\xi)+v(\xi) \text {. }
$$

If we choose $\xi$ where $v$ takes its maximum, we then obtain

$$
P_{p / q+}^{\prime \prime}(\xi) \geq v(\xi) \geq C_{r}^{\prime \prime} \varepsilon^{r+1} / q^{\gamma(r)} \text {. }
$$

Recall that $C_{r}^{\prime \prime}=C_{r}\left(C_{r} / 2 \theta^{\prime}\right)^{r+1}$ depends only on $r$ and $\theta^{\prime}=\theta+1$, and $\gamma(r)=$ $(r+1)^{2}$. In particular, $C_{r}^{\prime \prime}$ and $\gamma(r)$ are independent of the choice of $p / q$. In the case that $\omega$ is Liouville, we can choose $p / q$ so close to $\omega$ that

$$
C \theta^{\prime}|\omega q-p|<C_{r}^{\prime \prime} \varepsilon^{r+1} / q^{\gamma(r)},
$$

where $C$ is the constant in theorem 2.2 .

Suppose $\omega>p / q$. Then we perform the construction above, getting $h^{\prime \prime}$ which differs from $h$ in $C^{r+1}$ norm by $<\varepsilon$, with $P_{p / q+}^{\prime \prime}(\xi) \geq C_{r}^{\prime \prime} \varepsilon^{r+1} / q^{\gamma(r)}$. Moreover, if $\varepsilon$ is small enough, it is clear that $h^{\prime \prime}$ satisfies $\left(\mathrm{H}_{1}\right)-\left(\mathrm{H}_{5}\right)$ and $\left(\mathrm{H}_{6 \theta}\right)$. By theorem 2.2,

$$
P_{\omega}^{\prime \prime}(\xi) \geq P_{p / q+}^{\prime \prime}(\xi)-C \theta^{\prime}|\omega q-p| \geq C_{r}^{\prime \prime} \varepsilon^{r+1} / q^{\gamma(r)}-C \theta^{\prime}|\omega q-p|>0 .
$$

When $p / q<\omega$, we proceed in a similar way, making $P_{p / q-}^{\prime \prime}(\xi) \geq C_{r}^{\prime \prime} \varepsilon^{r+1} / q^{\gamma(r)}$. In either case we obtain $h^{\prime \prime}$ for which $P_{\omega}^{\prime \prime}$ does not vanish identically.

This proves theorem 2.1 .

\section{A problem}

Suppose $\omega \in \mathrm{DC}$. The infinum of the set of all $N$ for which there exists $C>0$ such that $|q \omega-p|>C q^{-N}$ for all $q, p \in \mathbb{Z} \backslash\{0\}$ is called the Diophantine exponent of $\omega$. The proof of theorem 2.1 in $\$ 5$ shows that for every positive integer $r$ there is a number $\gamma$ such that if $\bar{f} \in \mathscr{T}^{\infty}, f$ is its lift to the universal cover, and $\omega$ has Diophantine exponent $\geq \gamma$, then in any $C^{r}$ neighbourhood of $f$ in $\tilde{T}^{\infty}$ there is a $g$ such that $\bar{g}$ has no homotopically non-trivial invariant circle $\Gamma$ such that $\rho(g, \Gamma)=\omega$. (Here $\bar{g}$ is the unique element of $\mathscr{T}^{\infty}$ of which $g$ is the lift to the universal cover.) Let $\gamma(r)$ 
be the infinum of all such $\gamma$. Let $\alpha$ be the infinum of all positive numbers $a$ such that $\gamma(r)=\mathrm{O}\left(r^{a}\right)$ as $r \rightarrow \infty$. The proof of theorem 2.1 in $\S 5$ shows that $\alpha \leq 2$. On the other hand, KAM theory shows that $\alpha \geq 1$ (see e.g. Salamon [14]).

Problem. Find $\alpha$.

\section{Related results and problems}

Can one destroy many invariant circles by a $C^{\infty}$ small perturbation? Given $f \in \tilde{\mathscr{T}}^{\infty}$ and a countable set $\Sigma$ of Liouville numbers, one can find $g \in \tilde{T}^{\infty}$ arbitrarily close to $f$ in the $C^{\infty}$ topology such that there are no $\bar{g}$-invariant circles $\Gamma$ with $\rho(g, \Gamma) \in \Sigma$. It is enough to repeat the construction in $\$ 5$ countably many times.

J. Yoccoz has told us of the following result which he has obtained. Let $\bar{f} \in \mathscr{T}^{\infty}$ and let $K$ be the closure of the union of all homotopically non-trivial $C^{\infty} \bar{f}$-invariant circles. By a theorem of Birkhoff $[2, \S 3]$, every homotopically non-trivial $\bar{f}$-invariant circle is the graph of a Lipschitz function $u: \mathbb{R} / \mathbb{Z} \rightarrow \mathbb{R}$ with Lipschitz constant which depends only on the amount of twisting of $\bar{f}$. Consequently $K$ is the union of homotopically non-trivial $\bar{f}$-invariant circles. Let $\rho(f, K)$ denote the set of rotation numbers of such circles. By Birkhoff's theorem, $\rho(f, K)$ is a closed subset of $\mathbb{R}$. Yoccoz has shown that the set of Liouville numbers in $\rho(f, K)$ is residual in $\rho(f, K)$ in the sense of Baire category, by simple application of KAM theory.

Yoccoz's result suggests the following problem which we have been unable to resolve.

Problem. Let $\phi$ be a positive function, defined on the positive integers, and suppose $\phi$ grows faster than every polynomial. Let $\boldsymbol{A}$ denote the set of real numbers $\omega$ such that $|\omega-p / q|<\phi(q)^{-1}$ for infinitely many rational numbers $p / q$. Let $\mathscr{D}$ denote the set of $f \in \tilde{T}^{\infty}$ for which no homotopically non-trivial invariant circle has rotation number in $\boldsymbol{A}$. Is $\mathscr{D}$ dense in $\tilde{\mathfrak{T}}^{\infty}$ (with respect to the $C^{\infty}$ topology)?

Acknowledgements. I would like to thank Michel Herman for posing the problem I solve here, and for his hospitality in inviting me to give several talks on this and related results in his seminar at Palaiseau. I would like to thank Jürgen Moser and ETH, where part of this work was done, for the hospitality I have enjoyed during several extended stays, and for the opportunity to present these results in the Analysis Seminar at ETH. In addition, I would like to thank Stefan Demichelis, Charles Fefferman, Sergio Fenley, Hu Sen, David Rana and David Stuart for listening to oral presentations of various preliminary versions of this paper. I would also like to thank Charles Fefferman for pointing out a mistake in a proposed proof that $\mathscr{D}$ is dense. This paper was partially supported by NSF contract \#DMS-8504984.

\section{REFERENCES}

[1] V. Bangert. Mather sets for twist maps and geodesics on tori. Dynam. Rep. To be published.

[2] G. D. Birkhoff. Sur quelques courbes fermées remarquables. Bull. Soc. Math. France 60 (1932), 1-26. Reprinted in Collected Works, vol. II. New York (1950), 418-443.

[3] S. Bullett. Invariant circles for the piecewise linear standard map. Commun. Math. Phys. 107 (1986), 241-262. 
[4] A. Fathi. Appendix to Ch. I of [5].

[5] M. Herman. Sur les courbes invariantes par les difféomorphismes de l'anneau, vol. 1. Astérisque 103-104 (1983).

[6] M. Herman. Sur les courbes invariantes par les difféomorphismes de l'anneau, vol. 2. Astérisque 144 (1986).

[7] J. Mather. Non-existence of invariant circles. Ergod. Th. \& Dynam. Sys. 4 (1984), 301-309.

[8] J. Mather. A criterion for the non-existence of invariant circles. Publ. IHES 63 (1986), 153-204.

[9] J. Mather. More Denjoy minimal sets for area preserving diffeomorphisms. Comm. Math. Helv. 60 (1985), 508-557.

[10] J. Mather. Dynamics of area preserving mappings. Proc. ICM. To be published.

[11] J. Mather. Modulus of continuity for Peierls's barrier. Periodic Solutions of Hamiltonian Systems and Related Topics, ed. P. H. Rabinowitz et al. NATO ASI Series C 209. D. Reidel, Dordrecht (1987), 177-202.

[12] J. Moser. On invariant curves of area preserving mappings of an annulus. Nachr. Akad. Wiss. Göttingen, Math. Phys. Kl. (1962), 1-20.

[13] J. Moser. Stable and random motions in dynamical systems. Annals of Mathematical Studies 77. Princeton University Press (1973).

[14] D. Salamon. The Kolmogorov-Arnold-Moser theorem. Preprint. Forschungsinstitut für Mathematik, ETH Zürich (1986).

[15] C. Robinson. Generic properties of conservative systems. Amer. J. Math. 92 (1970), 562-603, 897-906.

[16] W. Rudin. Real and Complex Analysis. McGraw-Hill, New York (1966). 